\title{
Anica Betz, Caroline Schuttkowski, Linda Stark, Anne-Kathrin Wilms (Hrsg.): Sprache durch Dramapädagogik handelnd erfahren. Ansätze für den Sprachunterricht
}

\author{
Baltmannsweiler, Schneider Verlag Hohengehren 2016, 142 \\ Seiten. ISBN: 978-3-8340-1569-3
}

\section{Dragan Miladinovic}

Die in Buchrezensionen vertretenen Ansichten und Meinungen sind die der jeweiligen Rezensentinnen und Rezensenten und reflektieren nicht notwendigerweise die Position von SCENARIO.

Das vorliegende Buch liest sich wie ein Plädoyer für den Einsatz dramapädagogischer Methoden im fremd- und muttersprachlichen Unterricht. Die Dramapädagogik, so der Tenor, ebnet nicht nur mehrere Wege, Unterricht motivierender, emotionaler und erfolgreicher zu gestalten, sondern ist auch dazu geeignet, etwaige Hindernisse zu überwinden und Lernenden in der Sprach-, Literatur- und Kulturvermittlung neue und individualisierte Lernwege zu bieten. Der von Anica Betz, Caroline Schuttkowski, Linda Stark und AnneKathrin Wilms herausgegebene Sammelband gewährt seinen Leserinnen und Lesern Einblicke in sowohl theoretische Überlegungen als auch in die praktische Umsetzung von performativen Ansätzen im Unterricht, die Beiträge widmen sich vor allem der Rolle und dem Einsatz sowie dem Potenzial dramapädagogischer Methoden. Einigkeit besteht bei den Autorinnen und Autoren auch insofern, dass, obwohl die Dramapädagogik ein großes und vielschichtiges Potential innehat, sie dennoch in gewisser Weise ein Schattendasein fristet, und zwar sowohl in der Lehrendenausbildung ${ }^{1}$ als auch im Unterricht. Damit entgehe dem Bildungssektor eine großartige Gelegenheit, Unterricht zu gestalten, der Lernende nicht nur motiviert und emotional anspricht, sondern auch durch die in den Unterrichtsprozess integrierte Reflexionsphase seine volle Wirkung entfaltet.

Der Band steht in enger Verbindung zu einem Workshop zu Dramapädagogik und Grammatik, der an der Ruhr-Universität Bochum gehalten wurde. Dass Grammatik ein Hauptaugenmerk dieses Buchs ist, merken Leserinnen und Leser auch daran, dass die Herausgeberinnen in der Einleitung die Grammatikvermittlung besonders betonen. Außerdem nehmen sich darüber hinaus

\footnotetext{
${ }^{1}$ Vgl. in diesem Kontext die Untersuchung Performancekünste im Hochschulstudium von Fleiner (2016).
} 
zwei weitere Beiträge ebendiesem Thema an. Das Buch wurde schließlich mit Überlegungen zu anderen Domänen des Unterrichts, beispielsweise zum kulturellen Lernen, erweitert, sodass das vorliegende Manuskript entstand. Die Beiträge beginnen allesamt mit einer theoretischen Einleitung, gefolgt von praktischen Beispielen für die Umsetzung und/oder Praxisberichten, lediglich Manfred Schewe (63-77) tanzt sprichwörtlich aus der Reihe und beschäftigt sich mit einer terminologieorientierten Diskussion.

Den Fokus auf Grammatik legen Susanne Even (7-22) und Anna Brod (119-137). Even bezieht sich auf ihre Dissertation zur „Dramagrammatik“ (vgl. Even 2003) und zeigt, wie durch dramapädagogische Methoden Grammatikunterricht modifiziert werden kann. Durch eine Abfolge in sechs Phasen (8), die mit einer, wie weiter oben erwähnt, Reflexionsphase abschließt, werden „Lernwelten zugänglich [ge]macht, die über die Aneignung von Wissen über Fakten und Prozeduren herausgehen" (9). Der theoretischen Diskussion folgt eine praktische Umsetzung, an diese anschließend nimmt die Autorin einen möglichen Kritikpunkt selbst vorweg: Es gibt aktuell noch zu wenig empirische (Langzeit-)Studien, die belegen, inwiefern „,von einem definitiven (und nicht nur ,gefühlten') Zuwachs an Wissen und Können gesprochen werden kann“" (19).

Anna Brod (119-137) stellt in ihrem Beitrag einige von Expertinnen und Experten erarbeitete Methoden vor, die sie im Rahmen des Grammatikworshops an der Ruhr-Universität Bochum kennenlernte, und vergleicht sie miteinander. Der detaillierten Beschreibung der praktischen Umsetzung unterschiedlicher Grammatikthemen sowie der Reflexion als Teilnehmerin folgt abschließend eine Einordnung, u.a. nach Schwierigkeitsgrad und Gegenstand, der praktischen Beispiele in eine Tabelle. Im Schlussplädoyer verweist die Autorin ebenfalls auf einen möglichen Kritikpunkt: Dass „ein performativer Zugang zu Grammatik [...] rein kognitive Zugänge [wohl] nicht ersetzen, aber sinnvoll ergänzen“ (136) kann.

Dass Sprach-, Literatur- und Kulturdidaktik Hand in Hand mit nonverbaler Kommunikation gehen, darauf macht Carola Surkamp in ihrem Beitrag aufmerksam. Sie unterstreicht die Bedeutung von Einsatz, Wahrnehmung und Interpretation nonverbaler Phänomene für die Konstruktion von Bedeutung, aber auch, wie wichtig nonverbale Kommunikation für das interkulturelle Lernen ist. Trotz dieser Tatsache scheint das nonverbale Kommunizieren in der Fremdsprachendidaktik nach wie vor eine untergeordnete Rolle zu spielen. Das Weglassen dieser Dimension führe aber zu „künstliche[n] kommunikative[n] Situationen, die im starken Kontrast stehen zu lebensweltlichen Begegnungssituationen in der Fremdsprache" (28). Im Vordergrund stehe auch hier nicht das Vermitteln von Wissen, sondern eine Sensibilisierung für die körperliche Kommunikation. Gerade dafür eigne sich besonders die Dramapädagogik, so argumentiert die Autorin und demonstriert dies anhand von praktischen Beispielen für die Umsetzung im Unterricht.

Einblick in dramapädagogische Projekte bieten sowohl Franziska Elis (47-61) als auch Anastasia Moraitis (79-98) sowie Heike Mengele, Isabella Wlossek und 
Andreas Bülow (99-118). Elis stellt ihr Dissertationsprojekt vor, in dem sie sich für einen durch dramapädagogische Methoden unterstützten kontinuierlichen Übergang von der Primar- in die Sekundarstufe im Fach Englisch, aber auch darüber hinaus, einsetzt. Die aktuelle Diskrepanz in der Methodik der zwei Schultypen müsse überwunden werden, indem ein methodischer Grundstein in den vierten Klassen gelegt werde, auf den dann in der fünften Schulstufe aufgebaut werden könnte. Dazu müsse allerdings auch die Ausbildung von Lehrenden für die Primar- und auch Sekundarstufe hinterfragt werden.

Das sehr aktuelle Thema der Bildungsgerechtigkeit greift Moraitis' Beitrag auf. Die Autorin sieht kulturelle Bildung als „weitere, unabdingbare Schlüsselkompetenz für angestrebte Bildungsprozesse“ (79) und deren Grundstein sei spätestens in der Schule zu legen. Die Schichtzugehörigkeit entscheide viel mehr über den späteren Karriereerfolg als die Leistung, weswegen sie stark dafür plädiert, eine Lernumgebung zu schaffen, in der SchülerInnen „vielfältige Chance[n] erhalten, ihre individuellen Neigungen zu erkennen und auch selbstbestimmend auszubauen" (83). Damit könne der Bildungsungerechtigkeit entgegengewirkt werden und Schülerinnen und Schüler, an denen kulturelle Angebote normalerweise vorbeigehen, können auf diese Weise kulturelle Bildung erfahren. Dies könne unter Einsatz dramapädagogischer Methoden bewerkstelligt werden, da sie ein gruppenorientiertes und binnendifferenziertes Lernen ermöglichen. Anhand des Modellprojekts „Lampenfieber“ (ab 89) zeigt sie, wie die Ideen in die Tat umgesetzt werden können.

Mengele, Wlossek und Bülow (99-118) verweisen in ihrem Beitrag einerseits auf die Bedeutung der Dramapädagogik für das kulturelle Lernen, andererseits auf die Wichtigkeit der Einbeziehung von Herkunftssprachen im Sinne der Mehrsprachigkeit. Die Autorinnen sehen das Potenzial dramapädagogischer Methoden darin, einerseits interkulturelle Bildung, andererseits durch den gleichzeitigen Einbezug von Mehrsprachigkeit eine Anerkennung und Aufwertung der Herkunftssprachen und eine Identitätsstärkung zu ermöglichen. Anhand des Augsburger Projektseminars (107), bei dem ,Regelklassen' und getrennt unterrichtete ,Übergangsklassen' aufeinandertreffen, erläutern sie, inwiefern die von ihnen dargelegte Theorie praktisch umsetzbar ist.

Manfred Schewe (63-77) bietet im ersten Teil seines Beitrags einen fachgeschichtlichen Abriss der Dramapädagogik und wirft die Frage auf, inwiefern die britische Dramapädagogik fremdsprachenpädagogische Diskussionen in anderen Ländern beeinflusst hat und vice versa. Im zweiten Teil widmet er sich der in Fachdiskussionen häufig anzutreffenden Polarisierung zwischen Kunst und Wissenschaft, die sich letztlich auch in der aktuell verwendeten Terminologie widerspiegelt. Der Autor bezieht sich beispielhaft auf einige häufig als Gegensatzpaare konstruierte Begriffe (z.B. Prozess vs. Produkt), problematisiert das vorschnelle Konstruieren von Gegensätzen und gibt zu bedenken, dass „die Art und Weise, wie begrifflich gehandelt wird, fremdsprachpädagogische Wirklichkeit“" (63) schafft.

Der Sammelband ist besonders für Leserinnen und Leser, für die die Theorie und Praxis der Dramapädagogik, neu' ist, eine erste Anlaufstelle. Der Beitrag lässt 
sich aufgrund des Umfangs relativ schnell lesen und bietet einen grundlegenden theoretischen Überblick, den praktische Beispiele begleiten. Es werden aber auch versierte Dramapädagoginnen und -pädagogen sowohl Neues als auch Altes für sich (wieder)entdecken können, sodass sich die Lektüre für ein breites Publikum empfiehlt. Insgesamt ist dieser Band ein gelungenes Werk, dass nicht nur, wie der Titel verspricht, „Ansätze für den Sprachunterricht“, sondern auch über den Sprachunterricht hinausgehende Perspektiven bietet.

\section{Bibliografie}

Even, Susanne (2003): Drama Grammatik. Dramapädagogische Ansätze für den Grammatikunterricht Deutsch als Fremdsprache. München: iudicium

Fleiner, Micha (2016): Performancekünste im Hochschulstudium: Transversale Sprach-, Literatur- und Kulturerfahrungen in der fremdsprachlichen Lehrerbildung. Berlin: Schibri 